\title{
Development of size constancy in children: A test of the metacognitive theory
}

\author{
CARL E. Granrud \\ University of Northern Colorado, Greeley, Colorado
}

\begin{abstract}
Two studies investigated children's abilities to estimate the sizes of distant objects. Each included a size estimation task and a size-distance knowledge test, which assessed children's understanding of the effects of distance on objects' image sizes and perceived sizes. In Study $1(N=79$, age range $=5-10$ years $)$, high-knowledge children (those with above-median size-distance knowledge scores) made nearly accurate size estimates from a distance of $61 \mathrm{~m}$ and often reported using deliberate strategies to estimate size, whereas low-knowledge children underestimated size at this distance and typically reported no strategy use. In Study $2(N=60$, age range $=$ 6-11 years), high-knowledge children made nearly accurate size estimates from $61 \mathrm{~m}$ when given objectivesize instructions and underestimated size when given apparent-size instructions. Low-knowledge children underestimated size in response to both instruction sets. The results suggest that age-related changes in size estimation accuracy result from the development of cognitive abilities necessary for using deliberate strategies to supplement perception.
\end{abstract}

It has been well documented that children younger than 9 years of age underestimate objects' sizes beyond distances of about $15 \mathrm{~m}$, whereas 9- to 10 -year-old children and adults generally make nearly accurate size estimates when viewing distant objects (Brislin \& Leibowitz, 1970; Granrud \& Schmechel, 2006; Leibowitz, Pollard, \& Dickson, 1967; Zeigler \& Leibowitz, 1957). The causes of improvement in far-distance size estimation accuracy between early and later childhood have remained unknown, but several theories have been proposed to explain this developmental change. In light of the existing evidence (Granrud \& Schmechel, 2006; Leibowitz et al., 1967; Rapoport, 1967), the most plausible of these are the perceptual learning and metacognitive theories.

According to the perceptual learning theory (Leibowitz, 1974; Leibowitz et al., 1967), young children perceive the sizes of distant objects inaccurately because they are relatively insensitive to the visual cues, such as linear perspective, that provide information for object size when distances exceed about $15 \mathrm{~m}$. Children gradually learn to perceive distance and size from these cues, according to this theory, and are able to perceive the sizes of distant objects accurately by about 10 years of age.

The metacognitive theory, as proposed by Granrud (2004), represents a synthesis and extension of ideas expressed and implied in several sources, including Day (1987), Hamilton (1966), Leibowitz (1974), Rapoport (1967), and Wohlwill (1963). According to this theory, distant objects are misperceived by younger and older children and even adults: At all ages, distant objects are perceived as smaller than their actual sizes. Older children and adults, however, supplement perception with cognitive judgments that are based on knowledge about the effects of distance on perceived size. For example, when asked to estimate a distant object's size, they make nearly accurate size estimates, or often overestimate size (i.e., exhibit overconstancy), by using the distance compensation strategy, which involves deliberately inflating one's size estimate to compensate for the diminished perceived size of a distant object. In contrast, younger children respond to perceived size. As a result, they underestimate size (i.e., exhibit underconstancy). According to this theory, agerelated changes in size estimation accuracy result from the development of cognitive abilities, not from changes in perception.

Although studies have reported results consistent with the perceptual learning (Leibowitz et al., 1967) and metacognitive (Rapoport, 1967, 1969) theories, neither theory has been firmly supported, or refuted, by empirical research. The studies reported in this article tested the metacognitive theory.

\section{STUDY 1}

This study investigated the size estimation abilities of 5- to 10-year-old children. It had two parts: a size estimation task and a test that assessed participants' understanding of the effects of distance on image size and perceived size, which we referred to as the size-distance knowledge test. In the size estimation task, participants viewed standard objects one at a time at distances of 6.1 and $61 \mathrm{~m}$ and indicated which of nine nearby comparison objects

C. E. Granrud, carl.granrud@unco.edu 


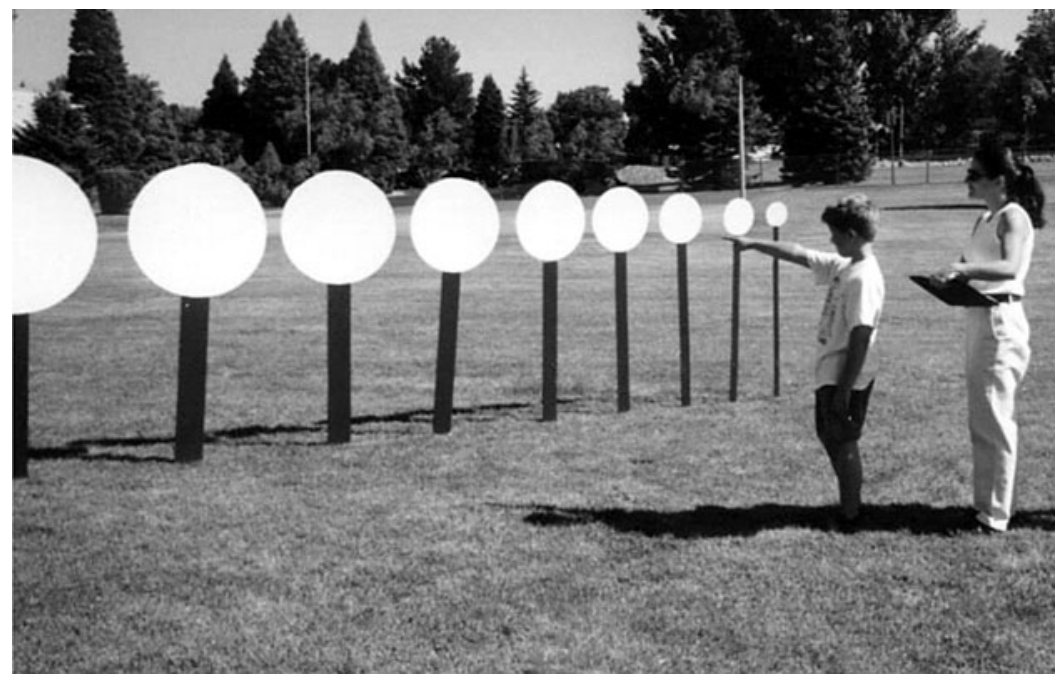

Figure 1. A research participant selects a comparison object to match the size of a distant standard object in the size estimation task (child's image used with parent's permission).

matched the standard objects in size (see Figure 1). After their response in the far-distance $(61 \mathrm{~m})$ condition, the participants were asked to explain why they selected the comparison object that they did. This was referred to as the size estimation follow-up question. At the beginning of the size estimation task, a pretest was conducted in which three standard objects were presented one at a time at a distance of $2.74 \mathrm{~m}$. This was done to ensure that all of the participants understood the task instructions and could accurately identify comparison objects that matched standard objects when the standard objects were nearby and their sizes were clearly perceptible.

The size-distance knowledge test included nine items in which the participants were asked about the effects of distance on image size and perceived size. In one item, for example, participants viewed a photograph of two equal-sized cars at different distances, in which the image of one car was much larger than that of the other. They were asked whether the cars were the same size or different sizes "in real life." They were then asked to explain their answers. A participant received points on each item for answers that displayed an understanding that image size decreases as distance increases, whereas physical size remains constant - saying, for example, that the two cars were the same size and that one looked larger because it was closer to the camera when the photograph was taken.

Only one previous study has investigated children's knowledge of the effects of distance on perceived size. Pillow and Flavell (1986) found that children as young as 3 years of age have some understanding that objects look smaller as their distances increase. Their results also suggested that this understanding improves between 3 and 4 years of age. Older children were not tested. The present study investigated 5- to 10-year-old children's knowledge about the effects of distance on image size and perceived size. We predicted that children would continue to accumulate knowledge about these effects after age 5 .

Four predictions about children's size estimation performances were made on the basis of the metacognitive theory. First, we predicted a correlation between sizedistance knowledge test score and size estimation accuracy in the far-distance $(61 \mathrm{~m})$ condition of the size estimation task. We reasoned that children who understood and could explain the effects of distance on image size and perceived size would be able to use the distance compensation strategy when judging the size of a distant object. Children who scored high on the size-distance knowledge test were, therefore, expected to exhibit nearly accurate size constancy, or overconstancy, in the far-distance condition, regardless of their ages. Children who scored low on the size-distance knowledge test were expected to exhibit underconstancy in the far-distance condition.

Second, we predicted no correlation between sizedistance knowledge score and size estimation accuracy at the near distance $(6.1 \mathrm{~m})$. On the basis of reports that children as young as 5 years of age estimate object size accurately at distances up to about $15 \mathrm{~m}$ (Brislin \& Leibowitz, 1970; Leibowitz et al., 1967; Zeigler \& Leibowitz, 1957), we expected that children would exhibit size constancy at the near distance, regardless of knowledge score.

Third, we predicted that children who scored high on the size-distance knowledge test would frequently report using the distance compensation strategy in the fardistance condition, whereas low scorers would typically report that they responded to perceived size. One previous study reported observations relevant to this prediction. Rapoport (1969) asked 5-, 7-, and 10-year-old children and adults to select the largest or smallest object from a set of five objects positioned at different distances up to $6.32 \mathrm{~m}$. The participants were also asked to describe 
how they had done the task. Adults tended to exhibit accurate size constancy and frequently said that they had used explicit strategies to judge size. The children tended to exhibit underconstancy and typically said that they had selected the objects on the basis of how they "looked." Although these observations are suggestive, no data on the participants' qualitative responses were presented in the Rapoport (1969) article. A goal of the present study was to collect data on children's reports of how they estimated size.

The fourth prediction concerned overconstancy. Overconstancy is sometimes exhibited by adults (e.g., Carlson, 1960, 1962; Gilinsky, 1955) and 9-year-old children (Granrud \& Schmechel, 2006) when they estimate the size of a distant object. As Wohlwill (1963) and others have pointed out, overconstancy reveals strategy use, because visual cues rarely indicate that a distant object is larger than its actual size. Overconstancy seems to result from research participants' making a cognitive adjustment that overcompensates for the diminished perceived size of the distant standard object. Since only high scorers on the size-distance knowledge test were expected to use strategies to estimate size, we predicted that overconstancy would be exhibited mainly by high scorers.

\section{Method}

Participants. Seventy-nine participants were included in the sample: 11 five-year-olds ( 5 boys and 6 girls), 14 six-year-olds ( 7 boys and 7 girls), 19 seven-year-olds ( 8 boys and 11 girls), 15 eight-yearolds ( 9 boys and 6 girls), 11 nine-year-olds ( 5 boys and 6 girls), and 9 ten-year-olds ( 7 boys and 2 girls). Fifteen additional participants were tested but not included in the sample because they did not pass the pretest (criteria for passing are described below). The participants were recruited from a university-operated elementary school and childcare center. A parent of each participant gave informed consent, and each participant gave verbal assent.

Size estimation task: Materials and Procedure. The size estimation task was conducted on a lawn adjacent to the participants' school. It consisted of three pretest trials and two test trials. On each trial, the participant viewed one standard object and nine comparison objects and was asked to point to the comparison object that matched the standard object in size. Although children of different ages generally viewed the objects from different heights (due to differences in standing height), Shallo and Rock (1988) and Granrud and Schmechel (2006) controlled for viewing height and found that age differences in size estimation cannot be attributed to this variable.

The comparison objects consisted of nine white disks, made from 1-cm-thick foamcore board, 76.2, 68.6, 61.0, 53.3, 45.7, 38.1, 30.5, 22.9 , and $15.2 \mathrm{~cm}$ in diameter. These sizes matched those used by Shallo and Rock (1988) and Granrud and Schmechel (2006). These objects were positioned to the participant's right, in a straight line parallel to the participant's line of sight when viewing the standard object, with the middle object $2.74 \mathrm{~m}$ from the participant. Each object was mounted on an upright, black wooden support, with its center $122 \mathrm{~cm}$ from the ground. Each support had a width equal to one fifth of the diameter of the object it supported.

There were nine standard objects for the pretest, which were identical to the comparison objects. Three standard objects were presented one at a time in the pretest. For each participant, one standard was chosen randomly from the three largest objects $(76.2,68.6$, and $61.0 \mathrm{~cm}$ ), one was chosen randomly from the three intermediatesized objects $(53.3,45.7$, and $38.1 \mathrm{~cm})$, and one was chosen randomly from the three smallest objects $(30.5,22.9$, and $15.2 \mathrm{~cm})$.
Each standard object was presented directly in front of the participant at a distance of $2.74 \mathrm{~m}$. There were three standard objects for the test trials, $61.0,53.3$, and $45.7 \mathrm{~cm}$ in diameter. Two test trials were conducted for each participant. In the near-distance trial, one standard was presented at a distance of $6.1 \mathrm{~m}$. In the far-distance trial, one standard was presented at a distance of $61 \mathrm{~m}$. The standard object was chosen randomly for each distance.

The pretest and test trials followed the same procedure. Before each trial, the participant looked away while the standard object was put into place. The participant was then instructed to turn around, inspect the standard, and point to the comparison object that was the same size. The participant's choice was recorded by two experimenters. If either experimenter could not determine which object had been selected, the participant was asked to walk to and touch the object. For the pretest, the three standard objects were presented in random order. On the test trials, the near- and far-distance trials were presented in counterbalanced order, with half of the children receiving the neardistance trial first and half receiving the far-distance trial first.

The size estimation follow-up question was asked immediately after the participants had made their size estimates in the far-distance test trial. The experimenter said, "You picked this circle [pointing to the comparison object that was selected] to match that one out there [pointing to the standard object]. Can you tell me why?" Responses were tape recorded and, when the participants had finished, the experimenter described any nonverbal gestures (such as pointing) that had been made.

Responses to the follow-up question were scored by three independent raters who were blind to the participants' results. Responses were placed into three categories on the basis of the rating given by at least two raters (at least two raters agreed for every response in the study). If the relationship between distance and perceived size was mentioned (e.g., "The circle looks smaller than it really is because it's far away"), the response was put into the strategy use category. If the participant said that the standard object looked the same size or was the same size as the selected comparison object, the response was placed into the object appearance category. If the participants reported that they did not know how they had done the task, said that they had guessed, or gave an explanation that did not fit into the first two categories, their responses were placed into the don't-know/ other category.

Three experimenters conducted the size estimation task. One interacted with the participants and recorded their responses. This experimenter's data were used in all the analyses. A second experimenter stood behind the first and independently recorded the participants' responses. A third put the standard objects in place. To prevent bias, the experimenters who recorded the responses were kept unaware of the standard object's size on each trial (and were instructed not to inspect the standard objects during testing). In addition, the experimenters were blind to the participants' size-distance knowledge test scores. The results recorded by the experimenters were highly correlated $(r=.99)$.

The participants were included in the sample if they chose the correct size match on at least two of the three pretest trials, and if any incorrect choice was made, the selected object was immediately adjacent to the correct object in the comparison object array. For the test trials, the dependent variable was percentage of error, computed as the diameter of the selected comparison object minus the diameter of the standard object, divided by the diameter of the standard object, times 100 .

Size-distance knowledge test: Materials and Procedure. The size-distance knowledge test was conducted after the size estimation task, on a separate day. It was administered by one experimenter, who was blind to the participants' size estimation task results, to each participant individually, indoors at the participants' school. It consisted of nine items, presented in random order for each participant.

Item 1 was modeled after a task used by Pillow and Flavell (1986). For Item $1 \mathrm{~A}$, the experimenter held up a toy dinosaur and asked two 
questions (in random order): "If I put this way far away over there [pointing out a window across the street], will it look big to you or will it look little to you?" and "If I put this right up close to your eyes, will it look big to you or will it look little to you?" The participants earned one point if they answered both questions correctly. For Item 1B, the experimenter asked, "You said that when this is far away (or close to your eyes) it will look little (or big). When it's far away (or close) is it really and truly little (big), or does it just look little (big)?" The participants received one point for answering both questions correctly.

Item 2 was modeled after a task used by Reith and Dominin (1997). The participants viewed a standard object (a white circle $23 \mathrm{~cm}$ in diameter, at a distance of $92 \mathrm{~cm}$ ) through a vertical pane of Plexiglas $(60 \times 40 \mathrm{~cm}$, at a distance of $24 \mathrm{~cm})$. Nine circles (the comparison objects) made of transparent pink acetate of various sizes $(2.5-10 \mathrm{~cm}$ in diameter) were affixed to the Plexiglas below the child's line of sight. The experimenter removed the $6-\mathrm{cm}$ pink circle from the Plexiglas, placed it on the Plexiglas in the participant's line of sight, and explained that it perfectly covered the standard circle (i.e., that the two objects were equal in projective size). The participants were then asked to predict which pink circle would cover the white circle if it were moved to four new distances (marked with vertical rods): two far distances (122 and $142 \mathrm{~cm}$ ) and two near distances (62 and $42 \mathrm{~cm}$ ). The predictions were made in pairs, so that each participant responded to the two far distances in succession and the two near distances in succession. The participants received one point for choosing a circle larger than $6 \mathrm{~cm}$ for the first near distance and a circle larger than the previous choice for the second near distance, and one point for choosing a circle smaller than $6 \mathrm{~cm}$ for the first far distance and a circle smaller than the previous choice for the second far distance.

Photographs were shown to the participants for Items 3-5. In each photograph, one object, or object part, was closer to the camera than another. The photograph for Item 3 was modeled after one in Rock (1975) and showed a woman sitting with the bottoms of her feet very close to the camera. Her feet looked very large. The photograph for Item 4, which appeared in Rock (1984), showed a fish hanging from a line held by a hand. A man standing at a greater distance had the same image size as the fish. In the photograph for Item 5, a hand positioned close to the camera appeared to be squeezing the head of an adult woman, who was standing farther away. In Part A of each item, the experimenter asked whether the woman had "really big feet or normal sized feet," whether the fish was "really big, as big as the man, or really small, like it would fit in a frying pan," and whether the woman's head was "really being squeezed by the hand or not really being squeezed by the hand." The participants received one point on each item for answering that the woman had normal sized feet, that the fish was small, and that the hand was not squeezing the woman's head. In Part B, the experimenter asked the participants to explain their answers in Part A. The participants received one point on each item for mentioning the relationship between distance and size (e.g., "Her feet are close, so they just look big").

For Items 6, 7, 8, and 9, photographs showed two objects at different distances that had equal objective sizes but very different image sizes. One photograph showed two girls (Item 6), one showed two cars (Item 7), one showed two steel towers that supported wires between them (Item 8), and one showed two white circles, similar to those used in the size estimation task (Item 9). For Part A of each item, the experimenter said, "This is a picture of two girls (or cars, electrical poles, etc.). In real life, are these two girls about the same size or very different sizes?" The participants received one point for saying that the girls (or cars, etc.) were about the same size. In Part B, the participants were asked to explain their answers. They received one point for mentioning the relationship between distance and size (e.g., "One girl looks smaller because she's farther away").

The participants' verbal responses were tape recorded and scored independently by three raters who were blind to the size estimation task results. The score for each item was determined by majority
Table 1

Pearson's $r$ Correlation Coefficients Between Age, Knowledge Score, Near Error, and Far Error in Study 1

\begin{tabular}{lccc}
\hline & $\begin{array}{c}\text { Knowledge } \\
\text { Score }\end{array}$ & Near Error & Far Error \\
\hline Age & $.64^{*}$ & .01 & $.39^{*}$ \\
Knowledge score & & .13 & $.63^{*}$ \\
Near error & & & $.33^{*}$ \\
\hline
\end{tabular}

*Significance at the .05 level.

(i.e., the score agreed upon by at least two of the three raters). Each item was worth up to 2 points, and 18 points was the maximum possible score.

\section{Results and Discussion}

Table 1 shows Pearson's $r$ correlations between age (in months), size-distance knowledge test score (referred to as knowledge score), percentage of error at the near distance (near error), and percentage of error at the far distance (far error). Near error was not correlated with knowledge score or age. Far error was significantly correlated with knowledge score, indicating that children who scored high on the size-distance knowledge test tended to make larger, more accurate, far-distance size estimates than those who scored low (the low scorers' errors were generally more negative, indicating greater underconstancy). Far error was also correlated with age, indicating that older children's far-distance size estimates tended to be larger, and more accurate, than those of younger children. Age was significantly correlated with knowledge score, indicating that older children generally scored higher than younger children on the size-distance knowledge test.

Partial correlation analyses revealed that knowledge score and far error were significantly correlated $[r(76)=$ $.55, p<.05]$ when the effects of age were partialed out. With the effects of knowledge score partialed out, age and far error were not correlated $[r(76)=-.03, p>.05]$. These results indicate that the correlation between age and far error was an artifact of the correlations between age and knowledge score and between knowledge score and far error. This suggests that the ability to make accurate far-distance size estimates does not appear at a specific age but, instead, appears when a child has acquired a requisite level of knowledge and/or reasoning skills.

An additional analysis examined the accuracy of size estimates made by children who scored high and low on the size-distance knowledge test. Forty participants scored 11 points or lower and were designated as the lowknowledge group. Thirty-nine scored 12 points or higher and were designated as the high-knowledge group. Table 2

Table 2

Mean Size Estimation Error in Each Condition in Study 1 (With Standard Deviations)

\begin{tabular}{|c|c|c|c|c|}
\hline \multirow{2}{*}{$\begin{array}{c}\text { Knowledge } \\
\text { Group }\end{array}$} & \multicolumn{2}{|c|}{ Near Error } & \multicolumn{2}{|c|}{ Far Error } \\
\hline & $M$ & $S D$ & $M$ & $S D$ \\
\hline Low $(n=40)$ & -10.73 & 9.87 & -21.15 & 16.00 \\
\hline $\operatorname{High}(n=39)$ & -10.13 & 10.59 & -0.87 & 16.85 \\
\hline
\end{tabular}




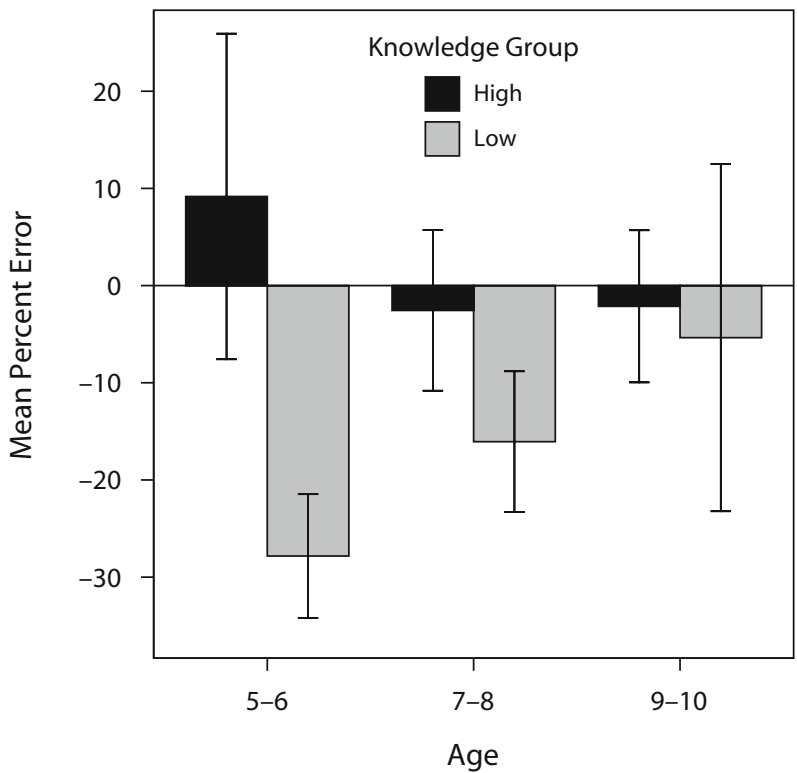

Figure 2. Mean far-distance size estimation errors by high- and low-knowledge children across ages in Study 1 . An error value of zero would indicate size constancy. Negative error values indicate underconstancy, and positive error values indicate overconstancy. Error bars represent $\pm 2 S E$.

shows mean size estimation errors for each group. Negative error values indicate underconstancy, positive values indicate overconstancy, and an error value of zero would indicate perfect constancy. For the high-knowledge group, mean near error differed significantly from zero $[t(38)=$ $-5.97, p<.01]$, but mean far error did not $[t(38)=-0.32$, $p>.05]$. These children, as a group, underestimated size at the near distance but exhibited nearly accurate size constancy at the far distance. For the low-knowledge group, mean error differed significantly from zero at the .01 level in both conditions $[t(39)=-6.87$ and -8.36 for near and far error, respectively]. These children exhibited underconstancy at both distances. As is shown in Figure 2, high-knowledge children generally made larger, more accurate far-distance size estimates than did low-knowledge children, regardless of age.

In the pretest, the mean number correct (out of three trials) was $2.59(S D=0.50)$ for the high-knowledge group and $2.50(S D=0.51)$ for the low-knowledge group. These means did not differ significantly $[t(77)=0.79, p>.05]$, indicating that the groups did not differ in their abilities to understand the task and make accurate size matches.

As is shown in Figure 3, the majority of participants who reported use of the distance compensation strategy exhibited size constancy or overconstancy at the far distance, whereas most of the participants who did not report strategy use (those whose responses fell in the object appearance or don't-know/other categories) exhibited underconstancy. As was predicted, a significant majority of the self-reported strategy users (21 out of 24) were in the high-knowledge group $\left[\chi^{2}(1, N=24)=13.50, p<.01\right]$. Also as was predicted, overconstancy was exhibited by significantly more high-knowledge (13) than low-knowledge (3) children $\left[\chi^{2}(1, N=16)=5.06, p<.05\right.$; Yates's correction was applied to this analysis due to low expected frequencies].

The following are examples of high-knowledge children's responses to the size estimation follow-up question. A 10-year-old who made an accurate size match said, "As things get farther away they look smaller, and since it looks about the same size as that one [pointing at a comparison object $43 \%$ smaller than the standard object], it would probably be a couple bigger: that one [pointing at the correct comparison object]," and a 6-year-old who exhibited overconstancy said, "It looked small only because it was far away." These reports seem to reveal deliberate use of the distance compensation strategy.

Children in the low-knowledge group typically said that they did not know how they had gone about the task or that they had responded on the basis of object appearance. For example, a 7-year-old said that she chose a comparison object $17 \%$ smaller than the standard object "because it looks the same size," and a 5-year-old chose a comparison object $43 \%$ smaller than the standard object because, she said, "they're the same size."

Two additional analyses were conducted. One showed no significant sex differences related to size-distance knowledge test scores or size estimation accuracy at the near or far distance. The other examined the participants' responses to the three standard objects in each condition of the size estimation task to test for response biases. Size estimates varied significantly in response to variations in the standard objects' sizes for both knowledge groups at both viewing distances, indicating that the children's size estimates did not result from a response bias, such as

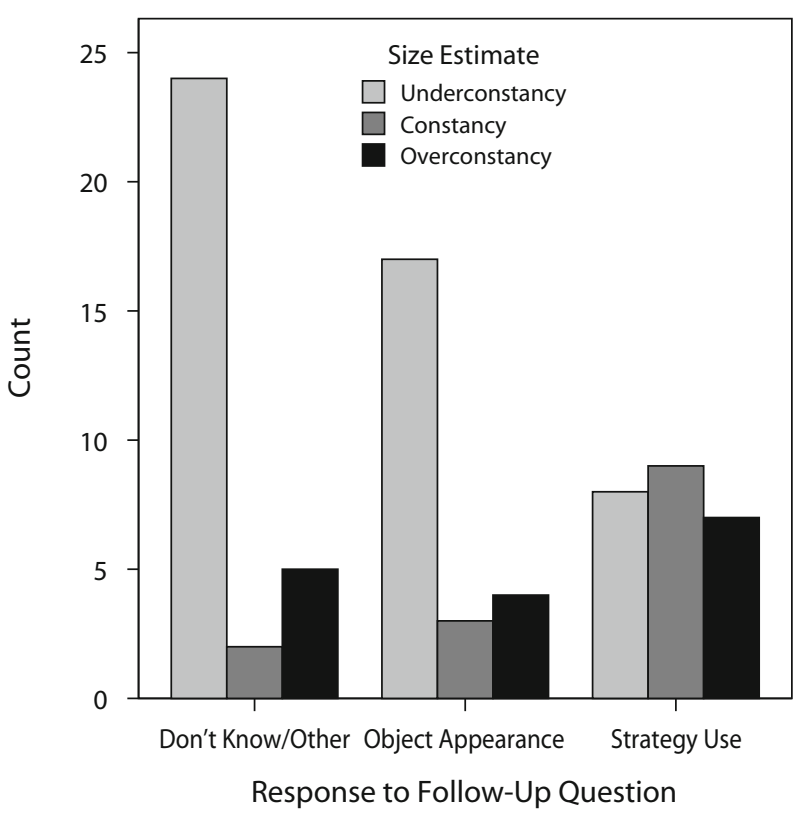

Figure 3. Number of Study 1 participants exhibiting underconstancy, size constancy, and overconstancy at the far distance, grouped by response to the size estimation follow-up question. 
choosing an object near the middle of the comparison object array without regard for the standard objects' sizes.

In sum, the results confirmed the three main predictions made from the metacognitive theory. Children who scored high on the size-distance knowledge test (i.e., those who understood and could explain the effects of distance on image size and perceived size) were more likely to report deliberate use of the distance compensation strategy when making far-distance size estimates, made more accurate far-distance size estimates, and were more likely to exhibit overconstancy than were children who scored low.

One result was unexpected. We anticipated that children would exhibit size constancy at the near distance, regardless of size-distance knowledge test score. But the children in both knowledge groups exhibited underconstancy at the near distance. This result confirms previous findings that 5- to 9-year-old children exhibit underconstancy at distances of 6-8 m (Beryl, 1926; Cohen, Hershkowitz, \& Chodack, 1958; Rapoport, 1967, 1969), and it conflicts with reports of accurate size constancy by 5 years of age at viewing distances up to about $15 \mathrm{~m}$ (Brislin \& Leibowitz, 1970; Leibowitz et al., 1967; Zeigler \& Leibowitz, 1957). Of primary importance for the present study, however, was the finding of no difference between the two groups' near-distance size estimates. If the high-knowledge children's more accurate far-distance size estimates resulted from higher levels of perceptual abilities, motivation, or task proficiency, they would be expected to make more accurate size estimates at the near distance as well. The results, therefore, suggest that the high- and low-knowledge group's different performances at the far distance did not result from differences in any of these variables. The groups' similar pretest results also support this conclusion.

\section{STUDY 2}

Study 2 investigated the effects of instructions on children's size estimates. Two sets of instructions were given in the size estimation task: apparent-size and objectivesize instructions. The apparent-size instructions directed the participants to choose a comparison object that matched the size that the standard object appeared to be, regardless of its actual size. The objective-size instructions directed them to choose a comparison object that matched the standard object's actual size, regardless of its perceived size.

Adults' far-distance size estimates are influenced by instructions. Although a variety of viewing distances, stimulus objects, and size estimation methods have been used in studies with adults and varying results have been obtained, adults consistently make larger size estimates when given objective-size instructions than when given apparent-size instructions (Carlson, 1960, 1962; Epstein, 1963; Leibowitz \& Harvey, 1967, 1969).

The effects of instructions on children's size estimates have also been investigated previously. Using viewing distances of 7-9 m, Rapoport (1967) found that 10- to 20 -year-olds made larger size estimates when given objective-size instructions than when given apparent-size instructions, whereas 5-, 7-, and 9-year-olds exhibited similar degrees of underconstancy in responses to both types of instructions. Rapoport (1967) concluded from these results that changes in size estimation performance between early childhood and adulthood result from the development of cognitive abilities and not from changes in perception.

Although Rapoport's (1967) findings are consistent with the metacognitive theory, she later pointed out that "the wording of a given set of instructions cannot be presumed to insure a homogeneous approach to the task across ages" (Rapoport, 1969, p. 366). Making a similar point, Day (1987) noted that the younger children in the Rapoport (1967) study may not have understood the difference between the two types of instructions. If this were the case, these children would not be expected to make different size estimates in response to the two instruction sets.

In Study 2, a pretest was conducted to determine whether the children understood, and responded appropriately to, the apparent- and objective-size instructions. In the pretest, the participants viewed a circle that was alternately placed under a convex lens, which magnified its image, and a concave lens, which reduced its image size. They were asked to select, from a set of comparison circles, a circle that matched the perceived size of the circle under the lens (apparent-size instructions) and one that matched its actual size (objective-size instructions). The same instructions were given in the pretest and the size estimation task (with minor modifications necessary to make the instructions appropriate for each task). If a participant passed the pretest, it indicated that he or she understood and could respond appropriately to the two instruction sets used in the size estimation task.

Four predictions were made on the basis of the metacognitive theory and the results of Study 1. The first three referred to the far-distance condition of the size estimation task. First, we predicted that high-knowledge children would make nearly accurate size estimates when given objective-size instructions and would underestimate size when given apparent-size instructions, whereas lowknowledge children would underestimate size in response to both instruction sets. Second, we predicted that highknowledge children would report that they had used the distance compensation strategy when given objective-size instructions and would report that they had responded to perceived size when given apparent-size instructions, whereas low-knowledge children would report that they had responded to perceived size regardless of the instructions given. Third, we predicted that overconstancy would be exhibited primarily by high-knowledge children in response to objective-size instructions. Finally, we predicted that near-distance size estimates would be unaffected by instructions and unrelated to size-distance knowledge test scores. 


\section{Method}

Participants. Sixty participants were included in the sample: 10 six-year-olds ( 6 boys and 4 girls), 11 seven-year-olds ( 6 boys and 5 girls), 11 eight-year-olds ( 5 boys and 6 girls), 8 nine-yearolds ( 4 boys and 4 girls), 9 ten-year-olds ( 1 boy and 8 girls), and 11 eleven-year-olds ( 4 boys and 7 girls). Four participants were tested but excluded from the sample for not passing the pretest (criteria for passing are described below). The participants were recruited from an after-school program for school-age children. A parent of each participant gave informed consent, and each participant gave verbal assent.

Pretest: Materials and Procedure. In the pretest, the participant viewed two identical standard circles, $2.5 \mathrm{~cm}$ in diameter, and five comparison circles, $1.5,2.0,2.5,3.25$, and $4.0 \mathrm{~cm}$ in diameter. All of the circles were printed in black ink on white paper. The standard circles were printed side by side, separated by $3 \mathrm{~cm}$, on a sheet $12 \mathrm{~cm}$ wide and $9 \mathrm{~cm}$ high, which lay flat in a box that was $12 \mathrm{~cm}$ wide and $9 \mathrm{~cm}$ high, with sides $5 \mathrm{~cm}$ deep. The comparison circles were centered on a sheet $28 \mathrm{~cm}$ wide and $21.5 \mathrm{~cm}$ high, with $1.5 \mathrm{~cm}$ separating each circle from its neighbors, and were arranged in order of size, with the largest circle on the left and the smallest on the right. Lenses were embedded in lids that fit over one half of the box. Two lenses were used: a convex lens that magnified image size by $30 \%$ and a concave lens that reduced image size by $30 \%$. Each lens was approximately circular in shape and approximately $5 \mathrm{~cm}$ in diameter. During each trial, one lid was placed on the right side of the box, which positioned a lens over the standard circle on the right. The standard circle on the left remained uncovered and visible throughout the pretest. The box containing the standard circles was placed on a tabletop at a distance of about $30 \mathrm{~cm}$ from the participant's eyes. The comparison circles were placed on the table next to the box, with the box between the participant and the comparison circles.

At the beginning of the pretest, the standard circles were presented without a lens in place, and the experimenter pointed out that they were exactly the same size. The participant was then asked to point to the comparison circle that matched the standard circles. If the participant responded correctly, testing proceeded. The experimenter explained the task as follows. "I'm going to put a lens over one of these circles, and I'm going to ask you two different questions about the circle. I'm going to ask you how big it looks and how big it really is." Four trials were then conducted, two with each lens.

Apparent-size instructions were given in one trial with each lens. In these trials, the participant was asked to point to a comparison circle that matched the size that the standard circle appeared to be, without regard to its actual size. A response was considered correct if the participant chose a circle larger than $2.5 \mathrm{~cm}$ on trials with the convex (magnifying) lens, and smaller than $2.5 \mathrm{~cm}$ on trials with the concave lens. Objective-size instructions were given for the other trial with each lens. On these trials, the participant was asked to point to the comparison circle that matched the actual size of the standard circle under the lens. A response was considered correct if the participant chose the $2.5-\mathrm{cm}$ comparison circle. The instructions were the same as those used in the size estimation task (the wording is given below, in the section describing the size estimation task), with minor modifications to make the instructions appropriate for the pretest.

The lens and instruction set given on the first trial were chosen randomly for each participant. The same lens was then used on the second trial, and the other instruction set was given. The other lens was used on the third and fourth trials; and the instruction set given on the third trial was chosen randomly. The participants were included in the sample if they answered correctly on all four pretest trials.

Size estimation task: Materials and Procedure. The size estimation task used the same materials and procedures as those in Study 1, with three exceptions. First, the pretest conducted in Study 1 was omitted. The pretest had been done to ensure that the children understood the size estimation task and could make accurate sizematching responses. However, the results from the near-distance condition provided evidence of comparable task proficiency in the high- and low-knowledge children. The Study 1 pretest was, therefore, unnecessary in Study 2, since near-distance conditions were included that controlled for task proficiency.

Second, a viewing distance of $5 \mathrm{~m}$ was used in the near-distance conditions in Study 2, as compared with $6.1 \mathrm{~m}$ in Study 1. The finding that most children exhibited underconstancy at the near distance in Study 1 was unexpected, and the 5-m distance was used to investigate whether children would exhibit size constancy at a nearer viewing distance.

Third, the size estimation task in Study 2 included four test trials: two at the near distance $(5 \mathrm{~m})$ and two at the far distance $(61 \mathrm{~m})$. At each distance, apparent-size instructions were given on one trial and objective-size instructions were given on the other. The condition (near-objective, near-apparent, far-objective, or far-apparent) given on the first trial was chosen randomly for each participant. On the second trial, the same distance as that of the first was used, and the other instruction set was given. The other viewing distance was then used for the third and fourth trials; and the instruction set given on the third trial was chosen randomly. A size estimation follow-up question was asked on each far-distance trial after the participant gave a response. For each trial, the standard object was chosen randomly from three possible objects (61.0, 53.3 , and $45.7 \mathrm{~cm}$ ).

At the beginning of the first trial, Experimenter 1 explained the task to the participant in the following way.

We're going to look at some circles out there [pointing to Experimenter 2, who was standing where the first standard object would be placed], and I'm going to ask you to compare each one to these circles over here [pointing to the comparison objects]. I'm going to ask you two different questions about the circles out there. I'm going to ask you how big they look and how big they really are. OK?

If the participant indicated understanding, verbally or by nodding, testing began.

The objective-size instructions were given as follows.

Look at that circle out there [pointing to the standard object] and look at these circles over here [pointing to the comparison objects]. I want you to point to the circle over here [pointing to the comparison objects] that is the exact same size as that circle out there [pointing to the standard]. Try to pick one that is exactly the same size if you measured them with a ruler. They may look the same size or they may not. That doesn't matter. What's important is that they really and truly are the same size.

The apparent-size instructions were given as follows.

Look at that circle out there [pointing to the standard object] and look at these circles over here [pointing to the comparison objects]. I want you to point to the circle over here [pointing to the comparison objects] that looks the same size as that circle out there [pointing to the standard]. It may really be the same size or it may not. That doesn't matter. What's important is that the circle out there [pointing] looks the same size as the circle you point to over here [pointing].

As in Study 1, the participants' size estimates were recorded independently by two experimenters. To prevent bias, the experimenters were kept unaware of the standard object used in each trial and were blind to the participants' size-distance knowledge test scores. Good agreement was found between the results recorded by the two experimenters $(r=.99)$.

Size-distance knowledge test: Materials and Procedure. The size-distance knowledge test used the same materials and procedures as those in Study 1, with two exceptions. First, this test was conducted before the size estimation task in Study 2. Second, Item 2 was omitted. The test, therefore, consisted of eight items, and had a maximum score of 16 points. Item 2 was the most time-consuming 
Table 3

Pearson's $r$ Correlation Coefficients Between Age, Knowledge Score, and Size Estimation Error in Each Condition in Study 2

\begin{tabular}{lcccccc}
\hline & & \multicolumn{2}{c}{ Near Error } & & \multicolumn{2}{c}{ Far Error } \\
\cline { 3 - 4 } \cline { 7 - 8 } & Knowledge & Objective & Apparent & & Objective & Apparent \\
\hline Age & $.63^{*}$ & .12 & -.06 & & $.26^{*}$ & -.11 \\
Knowledge & & .09 & .10 & & $.36^{*}$ & -.07 \\
\hline
\end{tabular}

*Significance at the .05 level.

Table 4

Mean Size Estimation Error in Each Condition in Study 2 (With Standard Deviations)

\begin{tabular}{|c|c|c|c|c|c|c|c|c|}
\hline \multirow{3}{*}{$\begin{array}{c}\text { Knowledge } \\
\text { Group }\end{array}$} & \multicolumn{4}{|c|}{ Near Distance } & \multicolumn{4}{|c|}{ Far Distance } \\
\hline & \multicolumn{2}{|c|}{ Objective } & \multicolumn{2}{|c|}{ Apparent } & \multicolumn{2}{|c|}{ Objective } & \multicolumn{2}{|c|}{ Apparent } \\
\hline & $M$ & $S D$ & $M$ & $S D$ & $M$ & $S D$ & $M$ & $S D$ \\
\hline Low $(n=29)$ & -12.84 & 9.66 & -16.17 & 10.14 & -16.95 & 18.75 & -22.12 & 19.58 \\
\hline $\operatorname{High}(n=31)$ & -11.14 & 10.62 & -12.63 & 11.08 & 0.59 & 23.53 & -25.02 & 21.43 \\
\hline
\end{tabular}

item, and omitting it allowed the test to be done more quickly and easily. The data from Study 1 were reanalyzed with the results from Item 2 omitted, and the same overall results were obtained.

\section{Results and Discussion}

Table 3 shows correlations between age (in months), size-distance knowledge test score, and percentage of error in the four conditions of the size estimation task. At the far distance, objective-size error was significantly correlated with knowledge score and age, indicating that children with higher knowledge scores tended to make larger, more accurate size estimates than did children with lower scores, and older children generally made larger, more accurate size estimates than did younger children. Far-distance, apparent-size error was not correlated with age or knowledge score. At the near distance, neither objective- nor apparent-size error was correlated with age or knowledge score. As in Study 1, age and knowledge score were correlated.

Partial correlation analyses showed that far-distance, objective-size error was correlated with knowledge score with the effects of age partialed out $[r(57)=.36, p<.05]$ but was not correlated with age with the effects of knowledge score partialed out $[r(57)=-.02, p>.05]$. These results replicated the Study 1 finding that far-distance size estimation accuracy (with objective-size instructions) is more closely related to knowledge score than to age.

As in Study 1, the sample was divided into two groups to examine the accuracy of size estimates made by low- and high-knowledge children. Twenty-nine children received scores of 12 points or lower and were designated as the lowknowledge group, and 31 received scores of 13 points or higher and were designated as the high-knowledge group. Table 4 shows mean percentage-of-error values for the two groups in the four conditions of the size estimation task. The low-knowledge group's percentage-of-error means differed significantly from zero at the .01 level in all four conditions $[t(28)=-7.16,-8.59,-4.87$, and -6.08 in the nearobjective, near-apparent, far-objective, and far-apparent conditions, respectively]. Instructions had no effect on this group's size estimates. They exhibited significant under- constancy at the near and far distances in response to both instruction sets. The high-knowledge group's percentageof-error mean did not differ from zero in the far-objective condition $[t(30)=0.14, p>.05]$ but differed from zero at the .01 level in the other conditions $[t(30)=-5.84$, -6.35 , and -6.50 in the near-objective, near-apparent, and far-apparent conditions, respectively]. Instructions affected this group's far-distance size estimates. These children exhibited nearly accurate size constancy when given objective-size instructions and significant underconstancy when given apparent-size instructions. These results indicate that high-knowledge children distinguish between a distant object's perceived size and actual size, whereas lowknowledge children do not. In addition, as can be seen in

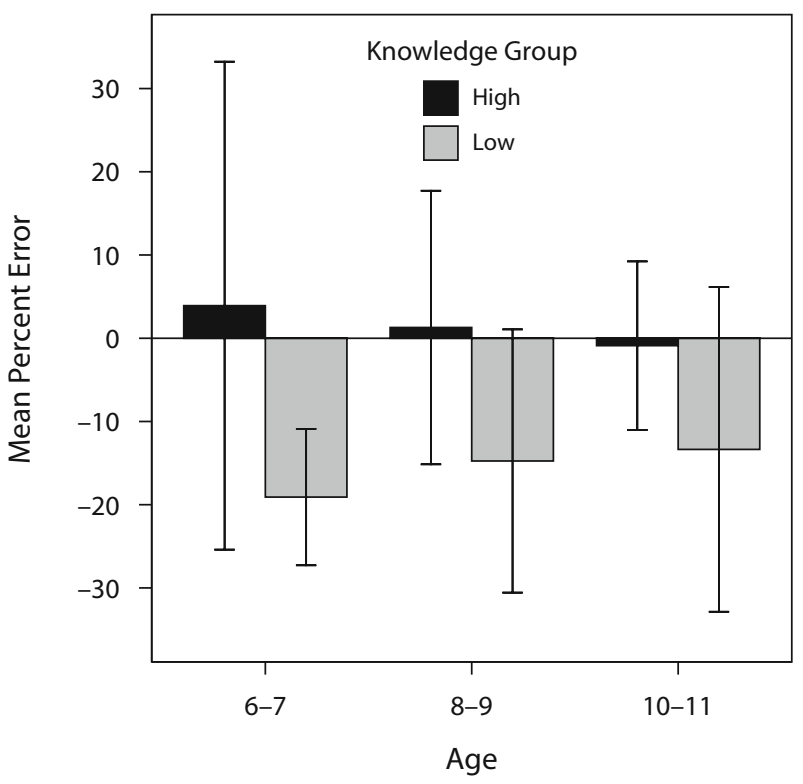

Figure 4. Mean far-distance, objective-size errors by high- and low-knowledge children across ages in Study 2. An error value of zero would indicate size constancy. Negative error values indicate underconstancy, and positive error values indicate overconstancy. Error bars represent $\pm 2 S E$. 


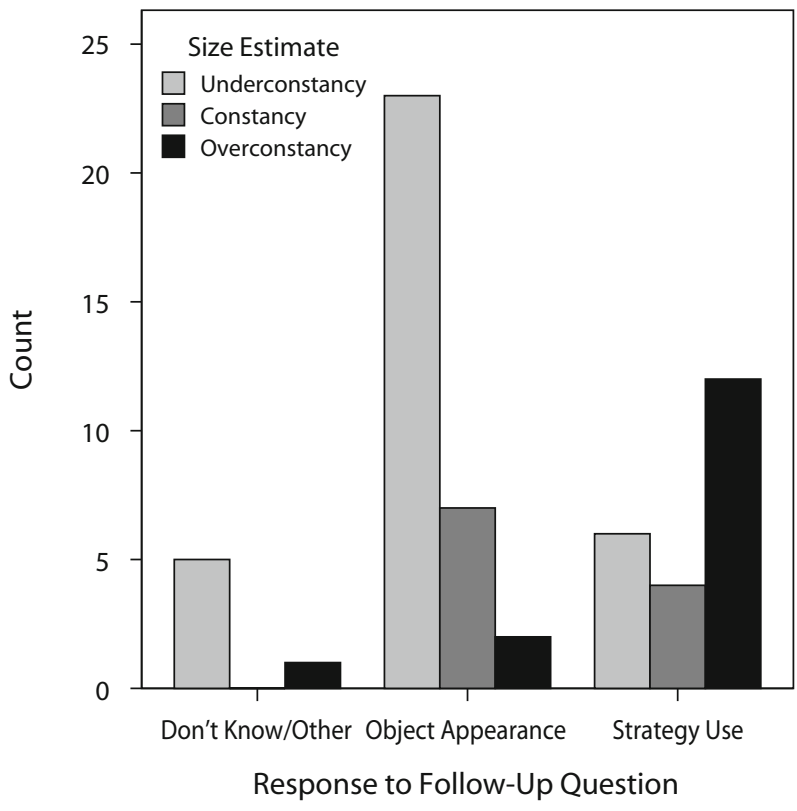

Figure 5. Number of Study 2 participants exhibiting underconstancy, size constancy, and overconstancy in the far-distance, objective-size condition, grouped by response to the size estimation follow-up question.

Figure 4, high-knowledge children generally made more accurate far-distance, objective-size estimates than did lowknowledge children, regardless of age.

As is shown in Figure 5, the majority of the participants who reported use of the distance compensation strategy in the far-distance, objective-size condition exhibited size constancy or overconstancy, whereas the majority of those reporting no strategy use exhibited underconstancy. As was predicted, a significant majority of the self-reported strategy users (20 out of 22) were in the high-knowledge group $\left[\chi^{2}(1, N=22)=14.72, p<.01\right]$. In the fardistance, apparent-size condition, the majority of high(30 out of 31) and low-knowledge (28 out of 29) children reported that they responded to object appearance, and the majority of those reporting object appearance responses exhibited underconstancy: 26 out of 30 in the highknowledge group and 25 out of 28 in the low-knowledge group. These results suggest that strategy use generally results in size constancy or overconstancy, whereas object appearance responses result in underconstancy.

Fifteen overconstancy responses occurred in the fardistance, objective-size condition, and a significant majority of these (12) were made by high-knowledge children $\left[\chi^{2}(1, N=15)=4.40, p<.05\right.$; Yates's correction was applied to this analysis due to low expected frequencies]. Overconstancy responses rarely occurred in the other conditions, and those that did were not related to knowledge scores.

As in Study 1, no differences were found between the high- and low-knowledge groups' near-distance size estimates. This suggests that the two groups did not differ in perceptual abilities, motivation, or task proficiency. Near- distance size estimates were not affected by instructions, indicating that neither the high- nor the low-knowledge children distinguished between perceived and objective size at $5 \mathrm{~m}$.

Two results suggest that the high-knowledge children's different responses to the two instruction sets cannot be attributed to demand characteristics. First, the order in which the instruction sets were given did not significantly affect size estimates at either distance. Second, this group's size estimates were not affected by instructions at the near distance, contrary to what would be expected if these children felt pressured to vary their size estimates in response to varying instructions.

It is important to note that every child in the sample answered correctly on all four trials of the pretest. When the standard circle was under a lens that reduced image size, for example, even the youngest children, and those who scored lowest on the size-distance knowledge test, identified the comparison circle that matched the standard circle's objective size, and they chose a circle smaller than the standard circle when asked to match its perceived size. This indicates that the children understood both sets of instructions and that the instructions elicited appropriate task attitudes in every child in the sample.

In sum, the results confirmed all of the predictions made from the metacognitive theory. In addition, Study 2 replicated all of the main results from Study 1. Although only one test trial was given in each condition in each study, the consistent findings of the two studies indicate that reliable results were obtained.

\section{GENERAL DISCUSSION}

Overall, the findings suggest that, when viewing distant objects, most 5- and 6-year-old children make no distinction between perceived and objective size, report no strategy use, and consistently underestimate objective size. By 9-10 years of age, most children distinguish between distant objects' perceived and objective sizes, make more accurate objective size estimates, and deliberately use the distance compensation strategy to judge the objective sizes of distant objects. This strategy does not necessarily result in perfect constancy but, instead, seems to involve a heuristic-based guess that can result in constancy, underconstancy, or overconstancy. The results further suggest that developmental changes in size estimation performance are more closely related to the abilities to understand and explain the effects of distance on image size and perceived size than to age. For example, 6- and 7-year-olds who scored high on the size-distance knowledge test generally made more accurate far-distance objective-size estimates, were more likely to report using the distance compensation strategy, and were more influenced by instructions than were 8-and 9-year-olds who scored low. Given the quasi-experimental nature of these studies, we cannot conclusively identify the causes of agerelated improvements in size estimation accuracy. But the metacognitive theory provides a parsimonious explanation for all of the results. 
In contrast, the perceptual learning theory, which proposes that developmental changes in size estimation accuracy result from increased sensitivity to visual cues, cannot explain at least four aspects of the results. First, on the basis of this theory, participants who estimate size accurately at farther distances would be expected to estimate size accurately at nearer distances as well. If an observer is sufficiently sensitive to visual cues to achieve size constancy at $61 \mathrm{~m}$, sensitivity must also be sufficient to achieve constancy at 5 or $6.1 \mathrm{~m}$. The finding that highknowledge participants made more accurate objectivesize estimates at the far distance than at the near distance cannot be explained by sensitivity to visual cues. Second, many of the participants who exhibited approximate size constancy at $61 \mathrm{~m}$ reported that they used the distance compensation strategy in making their size estimates. If the development of size constancy results from increased sensitivity to visual cues, observers who achieve size constancy at $61 \mathrm{~m}$ should do so on the basis of veridical perception, and strategy use should be unnecessary. Third, the perceptual learning theory cannot account for the effects of instructions found in Study 2. If accurate size constancy is achieved by detecting visual cues, observers who achieve size constancy should make no distinction between perceived and objective size. For these observers, perceived size should be veridical and equal to objective size. Finally, the perceptual learning theory cannot explain overconstancy, which often occurs in studies with adult participants (e.g., Carlson, 1960, 1962; Gilinsky, 1955; Granrud \& Schmechel, 2006) and was exhibited by many high-knowledge children in Studies 1 and 2. In sum, the results conflict with the perceptual learning theory but support the metacognitive theory.

Two conclusions can be drawn from the results of the near-distance conditions. First, the finding of no association between size-distance knowledge scores and near-distance size estimates suggests that the high- and low-knowledge groups did not differ in motivation, task proficiency, or sensitivity to visual cues. An advantage on any of these variables would provide an advantage at the near distance as well as at the far distance size. Since the far-distance results cannot be explained by any of these variables, it seems likely that the children's self-reports were valid and that the high-knowledge children's more accurate size estimates resulted from deliberate strategy use by this group, but not by the other children. Second, the results indicate that size perception is inaccurate at distances of 5 and $6.1 \mathrm{~m}$, even for 10- to 11-year-old children. The finding that varying instructions had no effect on near-distance size estimates further suggests that neither high- nor low-knowledge children are aware that objective size differs from perceived size at $5 \mathrm{~m}$.

When tested under the conditions used in Study 2, college-age adults also underestimate object size by about $10 \%$ at $5 \mathrm{~m}$, and their size estimates at this distance are uninfluenced by varying instructions. At $61 \mathrm{~m}$, adults' size estimates are influenced by instructions and do not differ from those of high-knowledge children, but they are less likely to report using the distance compensation strategy
(Granrud, Granrud, \& Arnall, 2003). An interesting question for future research is whether adults use this strategy automatically without being aware of it, or whether they achieve size constancy for distant objects through a process different from that used by high-knowledge children.

The results of both studies indicate that cognitive development plays a role in the development of size constancy, but the specific cognitive abilities involved remain unknown. One possibility is that the development of accurate far-distance size estimation depends, at least to some extent, on the acquisition of knowledge about the effects of distance on perceived size. Although most of the lowknowledge children could say that an object "looks small" when far away (for Item 1 of the size-distance knowledge test), and all understood that objective and perceived size could differ, as indicated by their Study 2 pretest results, they did not exhibit awareness of these effects when making their far-distance size estimates. Their answers to the size estimation follow-up questions, and their similar size estimates in response to apparent- and objective-size instructions, seemed to reveal the belief that perceived size was veridical at $61 \mathrm{~m}$. Learning that perception can be inaccurate at far distances and that strategies are needed to supplement perception may be the key development underlying agerelated changes in far-distance size estimation accuracy.

Alternatively, it is possible that young children understand the effects of distance on perceived size but lack the reasoning abilities needed to formulate and/or execute an effective strategy to compensate for the diminished perceived size of a distant object. Consistent with this hypothesis, Moore, Merriman, and Granrud (2007) recently found that reasoning ability, as measured by the Similarities and Block Design subscales of the WISC-III (Wechsler, 1991), is strongly correlated with 6- to 9-yearolds' far-distance size estimation accuracy, far-distance strategy use, and size-distance knowledge test performance. It remains unknown, however, whether advanced reasoning ability facilitates the acquisition of knowledge about the effects of distance on perceived size or allows children to formulate and/or execute strategies that apply this knowledge.

\section{AUTHOR NOTE}

The author thanks the ABC Child Development Center, the UNC Child Care Center, the University Lab School, Robin Anthony, and Marilyn Welsh for their help in recruiting participants; Melissa Granrud, April VonEitzen, Samantha Cooper, Meredith Joseph, Julia Koc, Jennifer Lewis, Elianna Mondragon, Shanti Pepper, Ryan Peterson, and Eric Warneke for their help in testing research participants; and Bill Merriman, Fred Owens, Jeremy Wolfe, and Michael Kavšek for their valuable comments regarding the manuscript. Correspondence concerning this article may be sent to C. E. Granrud, School of Psychological Sciences, University of Northern Colorado, Campus Box 94, Greeley, CO 80639 (e-mail: carl.granrud@unco.edu).

\section{REFERENCES}

BERYL, F. (1926). Über die Grossenauffassung bei Kindern. Zeitschrift für Psychologie, 100, 344-371.

BrisLin, R. W., \& Leibowitz, H. W. (1970). The effect of separation between test and comparison on size constancy at various age-levels. American Journal of Psychology, 83, 372-376. 
Carlson, V. R. (1960). Overestimation in size-constancy judgments. American Journal of Psychology, 73, 199-213.

CARLSON, V. R. (1962). Size constancy judgments and perceptual compromise. Journal of Experimental Psychology, 63, 68-73.

Cohen, W., Hershkowitz, A., \& Chodack, M. (1958). Size judgment at different distances as a function of age level. Child Development, 29, 473-479.

DAY, R. H. (1987). Visual size constancy in infancy. In B. E. McKenzie \& R. H. Day (Eds.), Perceptual development in early infancy: Problems and issues (pp. 67-91). Hillsdale, NJ: Erlbaum.

Epstein, W. (1963). Attitude of judgment and the size-distance invariance hypothesis. Journal of Experimental Psychology, 66, 78-83.

GiLINSKY, A. S. (1955). The effect of attitude upon the perception of size. American Journal of Psychology, 68, 173-192.

Granrud, C. E. (2004). Visual metacognition and the development of size constancy. In D. T. Levin (Ed.), Thinking and seeing: Visual metacognition in adults and children (pp. 75-90). Cambridge, MA: MIT Press.

Granrud, C. E., Granrud, M. A., \& Arnall, A. J. (2003, April). Effects of instructions on children's performances in a size constancy task. Paper presented at the meeting of the Society for Research in Child Development, Tampa, FL.

Granrud, C. E., \& Schmechel, T. T. N. (2006). Development of size constancy in children: A test of the proximal mode sensitivity hypothesis. Perception \& Psychophysics, 68, 1372-1381.

Hamilton, V. (1966). Size constancy and intelligence: A re-examination. British Journal of Psychology, 57, 319-328.

LeIBowitz, H. W. (1974). Multiple mechanisms of size perception and size constancy. Hiroshima Forum for Psychology, 1, 47-53.

Leibowitz, H. W., \& Harvey, L. O., JR. (1967). Size matching as a function of instructions in a naturalistic environment. Journal of Experimental Psychology, 74, 378-382.

Leibowitz, H. W., \& Harvey, L. O., JR. (1969). Effect of instructions, environment, and type of test object on matched size. Journal of Experimental Psychology, 81, 36-43.
Leibowitz, H. W., Pollard, S. W., \& Dickson, D. (1967). Monocular and binocular size matching as a function of distance at various age levels. American Journal of Psychology, 80, 263-269.

Moore, Z., Merriman, W. E., \& Granrud, C. E. (2007, April). Predictors of the use of the distance-adjustment strategy in children's size judgment. Paper presented at the meeting of the Society for Research in Child Development, Boston.

Pillow, B. H., \& Flavell, J. H. (1986). Young children's knowledge about visual perception: Projective size and shape. Child Development, 57, 125-135.

RAPOPORT, J. L. (1967). Attitude and size judgment in school age children. Child Development, 38, 1188-1192.

RAPOPORT, J. L. (1969). Size-constancy in children measured by a functional size-discrimination task. Journal of Experimental Child Psychology, 7, 366-373.

ReITH, E., \& Dominin, D. (1997). The development of children's ability to attend to the visual projection of objects. British Journal of Developmental Psychology, 15, 177-196.

Rock, I. (1975). An introduction to perception. New York: Macmillan.

Rock, I. (1984). Perception. New York: Freeman.

Shallo, J., \& Rock, I. (1988). Size constancy in children: A new interpretation. Perception, 17, 803-813.

WECHSLER, D. (1991). Wechsler Intelligence Scale for Children-third edition (WISC-III). San Antonio, TX: Psychological Corp.

WoHLWILl, J. F. (1963). The development of "overconstancy" in space perception. In L. P. Lipsitt \& C. C. Spiker (Eds.), Advances in child development and behavior (Vol. 1, pp. 265-312). New York: Academic Press.

Zeigler, H. P., \& Leibowitz, H. [W.] (1957). Apparent visual size as a function of distance for children and adults. American Journal of Psychology, 70, 106-109.

(Manuscript received September 5, 2008; revision accepted for publication October 28, 2008.) 